\title{
Prevalencia de cardiopatías congénitas diagnosticadas o tratadas por cateterismo cardíaco en pediatría
}

\author{
Prevalence of congenital heart disease diagnosed or treated by cardiac catheterism \\ in pediatrics
}

\author{
Lupo Méndez-Durán ${ }^{1}$, Rafael Echeverría-Consuegra², Osmar Pérez-Pérez", Víctor Barbosa-Sarabia, \\ Luz M. Contreras-Wilches ${ }^{1}$ y Karen Cañón-Ferreira ${ }^{1}$ \\ ${ }^{1}$ Universidad Metropolitana; ${ }^{2}$ Clínica General del Norte. Barranquilla, Colombia
}

\section{Resumen}

Objetivo: Determinar las cardiopatías congénitas más diagnosticadas a través de cateterismo cardíaco en una población pediátrica de la costa norte colombiana durante el período de 2007 a 2016 . Materiales y métodos: Estudio descriptivo, retrospectivo y multicéntrico, cuya población corresponde al total de pacientes de 0 a 18 años en quienes se realizó cateterismo cardíaco diagnóstico o terapéutico, o ambos, en diferentes ciudades de la costa norte colombiana en el período de 2007 a 2016. Para la tabulación de la información se utilizó el programa Epi Info 7.0. La muestra se integró con 4,245 pacientes. Resultados: El género predominante fue el femenino (53.5\%), con una media de edad de $3.9 \pm 4.9$ años y una mayor prevalencia de 1 a 11 meses de vida (40.6\%). Conclusiones: La comunicación interventricular fue la cardiopatía congénita más diagnosticada mediante cateterismo cardíaco (29.2\%), seguida por el conducto arterioso persistente (23.1\%), comunicación interauricular (11.7\%), tetralogía de Fallot (6.8\%), estenosis pulmonar (6.7\%), transposición de grandes vasos (3.5\%), atresia pulmonar con comunicación interventricular (2.5\%), canal AV total (2.4\%), doble salida ventricular derecha (2.4\%), coartación de aorta (1\%), insuficiencia mitral (0.98\%), atresia tricuspídea (0.96\%), atresia pulmonar sin comunicación interventricular (0.73\%), doble salida ventricular derecha (0.5\%) y otras malformaciones $(4.2 \%)$.

Palabras clave: Cardiopatías congénitas. Cateterismo cardíaco.

\section{Abstract}

Objective: To determine the most frequent congenital heart diseases diagnosed by cardiac catheterization in the pediatric population of the north coast of Colombia, during the period 2007 to 2016. Materials and methods: Descriptive, retrospective and multicentric study; the population corresponds to the total number of patients between 0 and 18 years of age in which diagnostic and therapeutic cardiac catheterization was performed in different cities of the Colombian north coast in the period 2007 to 2016. Epi Info 7.0 software was used for tabulation of information. Total sample was conformed of 4.245 patients.

\section{Correspondencia:}

*Lupo Méndez-Durán

E-mail: pediatria@unimetro.edu.co
Disponible en internet: 10-06-2021

Rev Colomb Cardiol. 2021;28(2):146-152

www.rccardiologia.com 0120-5633 / @ 2020 Sociedad Colombiana de Cardiología y Cirugía Cardiovascular. Publicado por Permanyer. Este es un artículo open access bajo la licencia CC BY-NC-ND (http://creativecommons.org/licenses/by-nc-nd/4.0/). 
Results: The sex mostly involved was the female with $53.5 \%$, a mean age of $3.9 \pm 4.9$ years, with a higher prevalence between 1 and 11 months of life with $40.6 \%$. Conclusions: The ventricular septal defect was the most frequent congenital heart disease diagnosed by cardiac catheterization with $29.2 \%$, followed in frequency by the persistent conducto with $23.1 \%$, the atrial septal defect $11.7 \%$, tetralogy of Fallot with $6.8 \%$, pulmonary stenosis with $6.7 \%$, transposition of large vessels $3.5 \%$, pulmonary atresia with ventricular septal defect $2.5 \%$, total AV channel $2.4 \%$, double right ventricular outlet $2.4 \%$, coarctation of aorta on $1 \%$, mitral insufficiency $0.98 \%$, tricuspid atresia $0.96 \%$, pulmonary atresia without ventricular septal defect $0.73 \%$, double right ventricular outlet $0.5 \%$ and other malformations $4.2 \%$.

Key words: Congenital heart disease. Cardiac catheterism.

\section{Introducción}

Las cardiopatías congénitas se definen como toda anomalía estructural del corazón o los grandes vasos'. Se producen por alteraciones de una estructura normal en el proceso embrionario y por falta de crecimiento del desarrollo embrionario en fases tempranas (tercera a décima semanas de gestación) ${ }^{2}$. Su etiología, aunque aún no es muy clara, reconoce tres factores participantes: genéticos, ambientales y la relación de éstos ${ }^{3}$.

Algunos estudios las describen como la malformación congénita más frecuente ${ }^{4}$; sin embargo, en fecha reciente, en un estudio en tres ciudades de Colombia (Bogotá, Ubaté y Manizales), las cardiopatías congénitas representaron la séptima malformación congénita más frecuente, precedida por las malformaciones de la oreja, el pie equino varo, la polidactilia, el síndrome de Down, el nevo, y el labio y paladar hendidos ${ }^{5}$; esto puede explicarse porque, en su mayor parte, las cardiopatías congénitas no se diagnostican al nacimiento, lo que debe preocupar en gran medida.

La incidencia registrada oscila entre $5 \%$ y $12 \% 6,7$, con variaciones dependientes de los criterios utilizados y los métodos diagnósticos; no obstante, se ha observado un aumento atribuido a la implementación de métodos diagnósticos para detección temprana y la búsqueda activa de estas malformaciones ${ }^{8}$.

Existen múltiples cardiopatías congénitas que desempeñan un papel importante en la salud de este país, debido a su gran complejidad en el diagnóstico y tratamiento; se ha publicado una incidencia de 15.73 / 10,000 nacidos vivos, con frecuencia hasta del $30 \%$ de todos los recién nacidos con malformaciones ${ }^{9}$. Se han descrito prevalencias de 7 y 8 por cada 1,000 recién nacidos, con una mortalidad del $3 \%$ y el $46 \%$ por malformaciones congénitas. La mortalidad es del $18 \%$ al $25 \%$ al primer año de vida y del $4 \%$ a los 16 años ${ }^{8,10}$.

En cuanto a la frecuencia de cardiopatías congénitas, diferentes autores han descrito la comunicación interventricular como la más común, seguida por la comunicación interauricular, el conducto permeable, la estenosis pulmonar, la coartación aórtica, la tetralogía de Fallot y la estenosis aórtica, la transposición de grandes arterias, el canal auriculoventricular y el síndrome del hemicardio izquierdo hipoplásico ${ }^{11,12}$. En este país, Baltaxe, et al. ${ }^{13}$ notificaron los defectos del tabique ventricular como las cardiopatías congénitas más frecuentes, con $27.3 \%$; otros defectos, entre ellos tetralogía de Fallot, defecto de tabicación auriculoventricular, DAP grande y doble salida del ventrículo derecho, representaron el 27.3\%; el ventrículo hipoplásico tuvo una frecuencia de $21.8 \%$, la anomalía de grandes arterias de $9.1 \%$, al igual que los defectos valvulares, y por último los defectos del tabique auricular de 5.5\%, todos diagnosticados por ecocardiograma. Peñaloza, en un estudio de corte transversal, en pacientes con indicación para ecocardiograma fetal por presencia de factores de riesgo o sospecha de anomalía en el ultrasonido obstétrico, encontró que de 99 ecocardiogramas fetales 40 se describieron como normales (40.4\%) y 56 como anormales (59.6\%), para un total de 130 hallazgos anormales: 33 anomalías septales, (25.3\%), 32 conotruncales (24.6\%), 27 de la unión AV derecha (20.7\%), 12 de la unión AV izquierda (9.2\%) y 7 trastornos de la conducción $(5.3 \%)^{14}$.

Por otra parte, en la costa norte de Colombia no existen registros de la frecuencia de cardiopatías congénitas de los cateterismos diagnósticos y terapéuticos realizados en los últimos años en la población pediátrica, por lo que es necesaria la realización de esta investigación.

\section{Materiales y métodos}

Estudio descriptivo, retrospectivo y multicéntrico, cuya población corresponde al total de pacientes entre 0 y 18 años en la que se realizó cateterismo cardíaco diagnóstico o terapéutico, o ambos, en diferentes ciudades de la costa norte colombiana en el período de 
2007 a 2016. La muestra total estuvo conformada por 4,245 pacientes. Se tomaron los datos de una fuente secundaria, el registro de pacientes intervenidos en 18 instituciones de salud de la costa norte de Colombia en el servicio de hemodinamia.

Se incluyó a pacientes pediátricos de 0 a 18 años, así como a individuos en quienes se efectuó cateterismo cardíaco diagnóstico o terapéutico y con datos completos de variables en estudio en la base de datos del procedimiento. Se excluyeron los datos incompletos de variables en estudio.

Los datos se tomaron de registros de cateterismos cardíacos practicados en instituciones especializadas de seis departamentos de la costa caribeña colombiana, los cuales se trasladaron a un formulario de recolección de la información; con posterioridad se realizó la tabulación en el programa Epi-Info 7.0 versión en español. El análisis estadístico incluyó métodos cuantitativos y se valoraron parámetros estadísticos descriptivos.

\section{Resultados}

Se obtuvo un total de 4,269 datos de pacientes en registros de cateterismos cardíacos realizados en instituciones especializadas de seis departamentos de la costa caribeña colombiana. De éstos se excluyó a 24 pacientes por criterios de selección y al final se presentaron los resultados de 4,245.

La muestra estudiada evidenció mayor prevalencia de cardiopatías congénitas en el sexo femenino, con $53.5 \%$ (Tabla 1). La distribución de acuerdo con la edad mostró que en el $40.6 \%$ de los pacientes el cateterismo se efectuó en el lapso de 1 a 11 meses de vida, con una media de $3.9 \pm 4.9$ años (Tabla 2).

La distribución, de acuerdo con enfermedades diagnosticadas por cateterismo, mostró que la de mayor prevalencia fue la comunicación interventricular con $29.2 \%$, proporción de la cual el $7.3 \%$ correspondió a una comunicación interventricular relacionada con enfermedad simple. Le siguió en prevalencia el conducto (23.1\%) vinculado con enfermedad simple $(4.9 \%)$, la comunicación interauricular (11.7\%), la tetralogía de Fallot $(6.8 \%)$, la estenosis pulmonar $(6.7 \%)$, la transposición de grandes vasos (3.5\%), la atresia pulmonar con comunicación interventricular $(2.5 \%)$, el canal AV total $(2.4 \%)$, la doble salida ventricular derecha $(2.4 \%)$, la coartación aórtica (1\%), la insuficiencia mitral $(0.98 \%)$, la atresia tricuspídea $(0.96 \%)$, la atresia pulmonar sin comunicación interventricular $(0.73 \%)$, la doble salida ventricular derecha $(0.5 \%)$ y otras
Tabla 1. Distribución de acuerdo con el género

\begin{tabular}{|l|c|c|}
\hline Género & $\mathbf{n}$ & $(\%)$ \\
\hline Femenino & 2,272 & $53.5 \%$ \\
\hline Masculino & 1,973 & $46.5 \%$ \\
\hline Total & 4,245 & $100 \%$ \\
\hline
\end{tabular}

Fuente: Registro de pacientes intervenidos por cateterismo.

Tabla 2. Distribución de acuerdo con la edad

\begin{tabular}{|l|c|c|}
\hline Edad & $\mathbf{n}$ & $\%$ \\
\hline$<1$ mes & 561 & $13.2 \%$ \\
\hline $1-11$ meses & 1,723 & $40.6 \%$ \\
\hline $1-5$ años & 797 & $18.8 \%$ \\
\hline$>5$ años & 1,164 & $27.4 \%$ \\
\hline Total & 4,245 & $100 \%$ \\
\hline
\end{tabular}

Fuente: Registro de pacientes intervenidos por cateterismo.

malformaciones (4.2\%), entre ellas anomalía parcial del retorno venoso pulmonar, isomerismos, anomalía de Epstein y ventrículo único izquierdo (Tabla 3).

La distribución de acuerdo con las enfermedades más frecuentes diagnosticadas por cateterismo, según el intervalo etario, reveló que en todos los grupos la de mayor prevalencia fue la comunicación interventricular, con $18.3 \%$ en menores de 1 mes, $35.3 \%$ en pacientes de 1 a 11 meses, $28.3 \%$ en pacientes de 1 a 5 años y $25.9 \%$ en mayores de 5 años (Tabla 4).

El cierre del conducto fue el procedimiento más practicado en los cateterismos terapéuticos, con $29.9 \%$, seguido en frecuencia por la valvuloplastia pulmonar con el $20.5 \%$ y el cierre de la comunicación interauricular con $13.6 \%$ (Tabla 5).

La distribución observada de acuerdo con los cateterismos por departamento mostró que en el Atlántico se realizó el $74.2 \%$ de éstos, seguidos en frecuencia por los departamentos de Cesar con $8 \%$, Magdalena con $6.9 \%$, Córdoba con 5\%, Bolívar con 3.8\% y Sucre con $2.1 \%$ (Tabla 6).

\section{Discusión}

Las cardiopatías congénitas de la niñez constituyen un reto para pediatras, cardiólogos pediatras, cirujanos cardiovasculares, intensivistas y neonatólogos. Los 
Tabla 3. Distribución de acuerdo con enfermedades diagnosticadas por cateterismo

\begin{tabular}{|c|c|c|}
\hline Enfermedades & n & $\%$ \\
\hline Comunicación interventricular & 930 & $21.9 \%$ \\
\hline $\begin{array}{l}\text { Comunicación interventricular relacionada } \\
\text { con enfermedad simple }\end{array}$ & 310 & $7.3 \%$ \\
\hline Conducto arterioso persistente & 771 & $18.2 \%$ \\
\hline Tetralogía de Fallot & 291 & $6.8 \%$ \\
\hline $\begin{array}{l}\text { Conducto relacionado con enfermedad } \\
\text { simple }\end{array}$ & 209 & $4.9 \%$ \\
\hline Estenosis pulmonar & 285 & $6.7 \%$ \\
\hline Comunicación interauricular & 499 & $11.7 \%$ \\
\hline Transposición de grandes vasos & 150 & $3.5 \%$ \\
\hline Canal auriculoventricular total & 101 & $2.4 \%$ \\
\hline $\begin{array}{l}\text { Atresia pulmonar con comunicación } \\
\text { interventricular }\end{array}$ & 110 & $2.5 \%$ \\
\hline Atresia tricuspídea & 41 & $0.96 \%$ \\
\hline Estenosis aórtica & 66 & $1.5 \%$ \\
\hline Coartación aórtica & 44 & $1 \%$ \\
\hline Insuficiencia mitral & 42 & $0.98 \%$ \\
\hline $\begin{array}{l}\text { Atresia pulmonar sin comunicación } \\
\text { interventricular }\end{array}$ & 31 & $0.73 \%$ \\
\hline Canal auriculoventricular parcial & 23 & $0.5 \%$ \\
\hline Doble salida ventricular derecha & 104 & $2.4 \%$ \\
\hline Sano & 58 & $1.4 \%$ \\
\hline Otras & 180 & $4.2 \%$ \\
\hline Total & 4,245 & $100 \%$ \\
\hline
\end{tabular}

nuevos métodos diagnósticos y el avance en las técnicas quirúrgicas han permitido mejorar los resultados y el pronóstico. Esta investigación fijó el objetivo de determinar las cardiopatías congénitas más frecuentes diagnosticadas por cateterismo cardíaco en la población pediátrica de la costa norte colombiana, durante el período de 2007 a 2016.

Se presentan los resultados de 4,245 pacientes en quienes se practicó cateterismo cardíaco en seis departamentos de la costa norte de Colombia; la mayor frecuencia de este procedimiento se observó en el Atlántico con $74.2 \%$, seguido por Cesar con el $8 \%$, Magdalena con el $6.9 \%$, Córdoba con el 5\%, Bolívar con el 3.8\% y Sucre con el 2.1\%. Debe entenderse que esta distribución no se vincula con una mayor prevalencia de cardiopatías en el departamento del Atlántico, sino con la mayor cantidad de entidades especializadas en el tratamiento de estas enfermedades. En tal sentido, Barranquilla es punto referente de la región.

En cuanto a las características de género, la mayor prevalencia se observó en el femenino con el 53.5\%, comportamiento que difiere del informe de Baltaxe, et al., ${ }^{13}$ un estudio conducido entre 2001 y 2005 en 11 hospitales de Bogotá, Cali, Manizales, La Mesa, Ubaté y Chiquinquirá que notificó una mayor frecuencia en el género masculino, con un $52.7 \%$.

Por otro lado, la edad media fue de $3.9 \pm 4.9$ años, con una mayor prevalencia entre 1 y 11 meses con el $40.6 \%$, respecto de los datos estadísticos de cateterismos realizados en la costa norte entre septiembre de 1997 y agosto de 2006. Se identifica un aumento de 12.3 puntos porcentuales en este intervalo, por lo que pasa a ser el intervalo más frecuente, probablemente explicable por una mayor prontitud diagnóstica y un mayor acceso al sistema de salud.

La distribución de enfermedades diagnosticadas por cateterismo mostró que la de mayor frecuencia fue la comunicación interventricular con el $21.9 \%$ y la relacionada con enfermedad simple con el $7.3 \%$, para un total de $29.2 \%$ de comunicación interventricular, seguida por conducto persistente con un $18.2 \%$ y su relación con anomalía simple en $4.9 \%$ para un total de $23.1 \%$, la comunicación interauricular con $11.7 \%$, la tetralogía de Fallot con $6.8 \%$ y la estenosis pulmonar con $6.7 \%$. Éstas fueron las cinco cardiopatías congénitas más frecuentes, las cuales, en conjunto, alcanzaron el $77.5 \%$ de todas las cardiopatías congénitas; este comportamiento es similar al que describieron Baspinar, et al., ${ }^{11}$ quienes notificaron que en niños nacidos en el Jordan University Hospital entre 2007 y 2014 la comunicación interventricular fue la cardiopatía congénita más diagnosticada, con un $43.1 \%$. Asimismo, el estudio de Baltaxe ${ }^{13}$ indicó que la comunicación interventricular era la cardiopatía de mayor frecuencia, con $27.3 \%$.

Con menor frecuencia, en esta serie se observaron la transposición de grandes vasos (3.5\%), la atresia pulmonar con comunicación interventricular $(2.5 \%)$, el canal AV total $(2.4 \%)$, la doble salida ventricular derecha $(2.4 \%)$, la coartación aórtica (1\%), la insuficiencia mitral $(0.98 \%)$, la atresia tricuspídea $(0.96 \%)$, la atresia pulmonar sin comunicación interventricular $(0.73 \%)$, la doble salida ventricular derecha $(0.5 \%)$ y otras malformaciones $(4.2 \%)$, entre estas últimas la anomalía parcial del retorno venoso pulmonar, isomerismos, 
Rev Colomb Cardiol. 2021;28(2)

Tabla 4. Distribución de acuerdo con enfermedades por grupo etario

\begin{tabular}{|c|c|c|c|c|c|c|c|c|}
\hline \multirow{2}{*}{$\begin{array}{l}\text { Edad } \\
\text { Afección }\end{array}$} & \multicolumn{2}{|c|}{$<1$ mes } & \multicolumn{2}{|c|}{ 1-11 meses } & \multicolumn{2}{|c|}{ 1-5 años } & \multicolumn{2}{|c|}{$>5$ años } \\
\hline & $\mathbf{n}$ & $\%$ & $\mathbf{n}$ & $\%$ & n & $\%$ & n & $\%$ \\
\hline Comunicación interventricular & 103 & $18.3 \%$ & 609 & $35.3 \%$ & 226 & $28.3 \%$ & 302 & $25.9 \%$ \\
\hline Conducto arterioso persistente & 67 & $11.9 \%$ & 551 & $31.9 \%$ & 134 & $16.8 \%$ & 228 & $19.5 \%$ \\
\hline Transposición de grandes vasos & 96 & $17.1 \%$ & 45 & $2.6 \%$ & 9 & $1.1 \%$ & 0 & $0 \%$ \\
\hline Comunicación interauricular & 8 & $1.4 \%$ & 84 & $4.8 \%$ & 178 & $22.3 \%$ & 229 & $19.6 \%$ \\
\hline Atresia pulmonar con comunicación interventricular & 34 & $6 \%$ & 26 & $1.5 \%$ & 16 & $2 \%$ & 34 & $2.9 \%$ \\
\hline Tetralogía de Fallot & 34 & $6 \%$ & 125 & $7.2 \%$ & 46 & $5.7 \%$ & 86 & $7.3 \%$ \\
\hline Canal auriculoventricular & 7 & $1.2 \%$ & 48 & $2.7 \%$ & 14 & $1.7 \%$ & 32 & $2.7 \%$ \\
\hline Estenosis pulmonar & 50 & $8.9 \%$ & 125 & $7.2 \%$ & 15 & $1.8 \%$ & 95 & $8.1 \%$ \\
\hline Otras & 162 & $29.2 \%$ & 110 & $6.8 \%$ & 129 & $20.3 \%$ & 158 & $14 \%$ \\
\hline Total & 561 & $100 \%$ & 1,723 & $100 \%$ & 797 & $100 \%$ & 1,164 & $100 \%$ \\
\hline
\end{tabular}

Fuente: Registro de pacientes intervenidos por cateterismo.

Tabla 5. Distribución de acuerdo con los cateterismos terapéuticos

\begin{tabular}{|l|c|c|}
\hline Procedimientos & $\mathbf{n}$ & $\%$ \\
\hline Cierre del conducto & 495 & $29.9 \%$ \\
\hline Valvuloplastia pulmonar & 339 & $20.5 \%$ \\
\hline Cierre de comunicación interauricular & 223 & $13.6 \%$ \\
\hline Angioplastia CoAo & 139 & $8.4 \%$ \\
\hline Auriculoseptostomía & 119 & $7.3 \%$ \\
\hline Cierre de comunicación interventricular & 92 & $5.5 \%$ \\
\hline Valvuloplastia aórtica & 76 & $4.6 \%$ \\
\hline Stent ductal & 72 & $4.4 \%$ \\
\hline Embolizaciones & 63 & $3.8 \%$ \\
\hline Otros & 33 & $2 \%$ \\
\hline Total & 1,651 & $100 \%$ \\
\hline Fuente: Registro de pacientes intervenidos por cateterismo. & \\
\hline
\end{tabular}

anomalía de Epstein y ventrículo único izquierdo. Estas menores frecuencias son similares a las de los datos estadísticos de cateterismos realizados en la costa norte entre septiembre de 1997 y agosto de 2006.

La distribución de las enfermedades más frecuentes de acuerdo con la edad mostró que en los menores de 1 mes, en orden descendente, las más comunes fueron la comunicación interventricular, la transposición de
Tabla 6. Distribución de acuerdo con cateterismos por departamento

\begin{tabular}{|l|c|c|}
\hline Departamento & $\mathbf{n}$ & $\%$ \\
\hline Atlántico & 3,150 & $74.2 \%$ \\
\hline Cesar & 340 & $8 \%$ \\
\hline Magdalena & 295 & $6.9 \%$ \\
\hline Córdoba & 212 & $5 \%$ \\
\hline Bolívar & 160 & $3.8 \%$ \\
\hline Sucre & 88 & $2.1 \%$ \\
\hline Total & 4,245 & $100 \%$ \\
\hline
\end{tabular}

Fuente: Registro de pacientes intervenidos por cateterismo.

grandes vasos, el conducto persistente y la estenosis pulmonar, algo similar a lo que describió Romero ${ }^{10}$ en una unidad neonatal entre 2011 y 2015. En el intervalo de 1 a 11 meses, la comunicación interventricular fue la más frecuente, seguida del conducto, la tetralogía de Fallot, la estenosis pulmonar y la comunicación interauricular; en el intervalo de 1 a 5 años fue igualmente la comunicación interventricular la más frecuente, seguida del conducto, la comunicación interauricular y la tetralogía de Fallot; por último, en el grupo de mayores de 5 años, la más frecuente fue la comunicación interventricular con el $25.9 \%$, seguida de la comunicación interauricular, el conducto y la estenosis pulmonar. 
El comportamiento de las cardiopatías congénitas respecto de la edad es similar al que publicó González, quien también notificó la comunicación interventricular como la de mayor prevalencia en todos los grupos etarios ${ }^{9}$.

De los 4,245 cateterismos realizados, 1,651 fueron terapéuticos, para una frecuencia de $38.6 \%$, y se observó un aumento de los cateterismos terapéuticos en comparación con el período de 1997 a 2006, cuya frecuencia fue del $14.1 \%$. El procedimiento efectuado con mayor frecuencia fue el cierre del conducto con el $29.9 \%$, seguido de la valvuloplastia pulmonar con $20.5 \%$, el cierre de la comunicación interauricular con $13.6 \%$, la angioplastia CoAo con $8.4 \%$, la auriculoseptostomía con $7.3 \%$, el cierre de la comunicación interventricular con $5.5 \%$, la valvuloplastia aórtica con $4.6 \%$, el stent ductal con $4.4 \%$, las embolizaciones con $3.8 \%$ y otros procedimientos con $2 \%$.

\section{Conclusiones}

Los datos llevan a concluir que la comunicación interventricular fue la cardiopatía congénita más diagnosticada por cateterismo cardíaco, con $29.2 \%$, seguida en frecuencia por el conducto persistente con $23.1 \%$, la comunicación interauricular con $11.7 \%$, la tetralogía de Fallot con $6.8 \%$, la estenosis pulmonar con $6.7 \%$, la transposición de grandes vasos con $3.5 \%$, la atresia pulmonar con comunicación interventricular con $2.5 \%$, el canal AV total con $2.4 \%$, la doble salida ventricular derecha con $2.4 \%$, la coartación aórtica con $1 \%$, la insuficiencia mitral con $0.98 \%$, la atresia tricuspídea con $0.96 \%$, la atresia pulmonar sin comunicación interventricular con $0.73 \%$, la doble salida ventricular derecha con $0.5 \%$ y otras malformaciones con el $4.2 \%$, como la anomalía parcial del retorno venoso pulmonar, los isomerismos, la anomalía de Epstein y el ventrículo único izquierdo.

Por otra parte, el género más afectado fue el femenino, con el $53.5 \%$, con una media de edad de $3.9 \pm 4.9$ años; la mayor prevalencia entre 1 y 11 meses de vida tuvo el $40.6 \%$, mientras que en todos los intervalos etarios la cardiopatía congénita más común fue la comunicación interventricular.

El departamento del Atlántico fue donde se practicó la mayor cantidad de cateterismos con $74.2 \%$, seguido por Cesar con el $8 \%$, Magdalena con $6.9 \%$, Córdoba con $5 \%$, Bolívar con $3.8 \%$ y Sucre con $2.1 \%$. Los cateterismos terapéuticos se efectuaron con una frecuencia de $38.6 \%$.
Se recomienda la publicación y divulgación de los resultados arrojados en esta investigación, ya que son los primeros conocidos en la costa caribeña de Colombia. De igual manera, se sugiere aportar esta información a los sistemas de vigilancia epidemiológica ya establecidos con el fin de idear políticas de salud pública para la región.

Por otra parte, conviene conducir estudios para comparar el diagnóstico ecocardiográfico anterior a la realización del procedimiento y confirmar si mostró concordancia con el diagnóstico del cateterismo, y, por último, llevar a cabo un seguimiento de la morbilidad y mortalidad de los pacientes intervenidos para determinar quiénes requirieron reintervención por complicaciones.

\section{Financiamiento}

Se dispuso de una financiación mixta; tanto los investigadores que participaron como también la Universidad Metropolitana proporcionaron financiamiento económico para la realización de la investigación, el diseño del estudio, la recolección, el análisis y la interpretación de los datos y la redacción del artículo.

\section{Conflicto de intereses}

Los autores declaran no tener conflicto de intereses.

\section{Responsabiliades éticas}

Protección de personas y animales. Los autores declaran que para esta investigación no se han realizado experimentos en seres humanos ni en animales.

Confidencialidad de los datos. Los autores declaran que en este artículo no aparecen datos de pacientes.

Derecho a la privacidad y consentimiento informado. Los autores declaran que en este artículo no aparecen datos de pacientes.

\section{Bibliografía}

1. Perich R. Cardiopatías congénitas más frecuentes y seguimiento en atención primaria. Pediatr Integral. 2012;XVI(8):622-35.

2. Maroto C, López MC. Guías de práctica clínica de la Sociedad Española de Cardiología en las cardiopatías congénitas del recién nacido. Rev Esp Cardiol 2001;54:49-66.

3. Jenkins KJ, Correa A, Feinstein JA, Botto L, Britt AE, Daniels SR, et al. Noninherited risk factors and congenital cardiovascular defects: current knowledge: a scientific statement from the American Heart Association Congenital Cardiac Defects Committee, Council on Cardiovascular Disease in the young: endorsed by the American Academy of Pediatrics. Circulation. 2007:115:2995-3014.

4. Villanueva Orbáiz R, Arráez Aviar LA. Epidemiología de las malformaciones congénitas y de sus factores de riesgo. Pediátrica. 2006; 26(9):308-15. 
Rev Colomb Cardiol. 2021;28(2)

5. Zarante I, Franco L, López C, Fernández N. Frecuencia de malformaciones congénitas: evaluación y pronóstico de 52.744 nacimientos en tres ciudades colombianas. Rev Biomédica Inst Nac de Salud. 2010; 30(1):78-84.

6. Pierpont ME, Basson CT, Benson DWJr, Gelb BD, Giglia TM, Goldmuntz E, et al. Genetic basis for congenital heart defects: current knowledge: a scientific statement from the American Heart Association Congenital cardiac Defects Committee, Council on Cardiovascular Disease in the young: endorsed by the American Academy of Pediatrics. Circulation. 2007;115:3015-38.

7. Feltes TF, Bacha E, Beekman RH $3^{\text {rd }}$, Cheatham JP, Feinstein JA, Gomes AS, et al. Indications for cardiac catheterization and intervention in pediatric cardiac disease. A scientific statement from the American Heart Association. Circulation. 2011;123:2607-52.

8. Ruiz JG, Romero R, Buitrago A, Granados C, Suárez F, Zarante I. Guía de práctica clínica. Detección de anomalías congénitas en el recién nacido. Ministerio de Salud y Protección Social - Colciencias. 2013;03:1-50.
9. González Y. Informe final del evento anomalías congénitas hasta el período epidemiológico 13 del año 2012. Inst Nac Salud. 2012;1-17.

10. Romero Y. Prevalencia de cardiopatías congénitas en la unidad neonatal de un hospital universitario de la ciudad de Bogotá entre 2011 y 2015. Universidad Colegio Mayor Nuestra Señora del Rosario. 2016;1-76.

11. Başpinar O, Karaaslan S, Oran B, Baysal T, Elmaci AM, Yorulmaz A. Prevalence and distribution of children with congenital heart diseases in the central anatolian region, Turkey. Turk J Pediatr. 2006;48:237-43.

12. Özkutlu S, Akça T, Kafali G, Beksaç S. The results of fetal echocardiography in a tertiary center and comparison of low-and high-risk pregnancies for fetal congenital heart defects. Anadolu Kardiyol Derg. 2010;10:263-9.

13. Baltaxe E. Garante I. Prevalencia de malformaciones cardíacas congénitas en 44,985 nacimientos en Colombia. Arch Cardiol Mex. 2006;76:263-8.

14. Peñaloza J, Prada S, Otero J. Prevalencia de hallazgos anormales en ecocardiogramas fetales en el Hospital Universitario de Santander, Bucaramanga (Colombia), 2007-2013. Rev Colm de Obst y Gin. 2015; $66(2) 110-5$ 